\title{
Singlet fission in pentacene dimers
}

\author{
Johannes Zirzlmeier ${ }^{a}$, Dan Lehnherr ${ }^{b}$, Pedro B. Coto ${ }^{c}$, Erin T. Chernick ${ }^{d}$, Rubén Casillas ${ }^{a}$, Bettina S. Basel ${ }^{a}$, Michael Thoss ${ }^{c}$, \\ Rik R. Tykwinski ${ }^{\mathrm{d}, 1}$, and Dirk M. Guldi ${ }^{\mathrm{a}, 1}$
}

\begin{abstract}
${ }^{a}$ Department of Chemistry and Pharmacy \& Interdisciplinary Center for Molecular Materials, Friedrich-Alexander-Universität Erlangen-Nürnberg, 91058 Erlangen, Germany; ${ }^{b}$ Department of Chemistry, University of Alberta, Edmonton, AB, Canada T6G 2G2; ' Institute for Theoretical Physics \& Interdisciplinary Center for Molecular Materials, Friedrich-Alexander-Universität Erlangen-Nürnberg, 91058 Erlangen, Germany; and ${ }^{\mathrm{d} D e p a r t m e n t}$ of Chemistry and Pharmacy \& Interdisciplinary Center for Molecular Materials, Friedrich-Alexander-Universität Erlangen-Nürnberg, 91054 Erlangen, Germany
\end{abstract}

Edited by David R. Reichman, Columbia University, New York, NY, and accepted by the Editorial Board March 9, 2015 (received for review November 26, 2014 )

Singlet fission (SF) has the potential to supersede the traditional solar energy conversion scheme by means of boosting the photonto-current conversion efficiencies beyond the $30 \%$ ShockleyQueisser limit. Here, we show unambiguous and compelling evidence for unprecedented intramolecular SF within regioisomeric pentacene dimers in room-temperature solutions, with observed triplet quantum yields reaching as high as $156 \pm 5 \%$. Whereas previous studies have shown that the collision of a photoexcited chromophore with a ground-state chromophore can give rise to SF, here we demonstrate that the proximity and sufficient coupling through bond or space in pentacene dimers is enough to induce intramolecular SF where two triplets are generated on one molecule.

acene oligomers | excited states | singlet fission | multireference perturbation theory | time-resolved spectroscopy

S inglet fission (SF) is a spin-allowed process to convert one singlet excited state into two triplet excited states, namely a correlated triplet pair (1). The ability to effectively implement SF processes in solar cells could allow for more efficient harvesting of high-energy photons from the solar spectrum and allow for the design of solar cells to circumvent the Shockley-Queisser performance limit (2). Indeed, several recent studies have demonstrated remarkably efficient solar cell devices based on SF (3-6).

One requirement that needs to be met to achieve SF is that the photoexcited chromophore in its singlet excited state must share its energy with a neighboring ground-state chromophore. As such, the potential of coupled chromophores to afford two triplet excited states via SF has been elucidated in, for example, a tetracene dimer with an SF yield of around 3\% $(3,7)$. Additionally, past experiments in single-crystal, polycrystalline, and amorphous solids of pentacene have documented that the efficiency of SF relates to the electronic coupling between these two chromophores $(8,9)$. Hence, molecular ordering in terms of crystal packing, that is, proximity, distances, orbital overlap, etc., is decisive with respect to gaining full control over and to finetuning interchromophoric interactions in the solid state $(10,11)$. Of equal importance are the thermodynamic requirements, namely that the energy of the lowest-lying singlet absorbing state must match or exceed the energy of two triplet excited states $\left(\mathrm{S}_{1} \geq 2 \mathrm{~T}_{1}\right)$ (11). In light of both aspects, hydrocarbons such as acenes - tetracene, pentacene, hexacene-and their derivatives are at the forefront of investigations toward a sound understanding and development of molecular building blocks for SF. In tetracenes, the singlet- and triplet-pair energy levels are nearly degenerate $\left(S_{1}=2 T_{1}\right)$, leaving no or little standard enthalpy of reaction for SF (12). In solution, the latter is, however, offset by sizable entropy rendering the process rather slow and, thus, inefficient (13). In addition, the low SF yield relates to the dimer geometry. Its nature hinders electronic coupling through space, leaving only through-bond coupling effective. The latter is, however, insufficient to enhance the SF rate $(7,14)$. In stark contrast, the relaxed triplet excited state in pentacenes has significantly less than half the energy of the singlet excited state. In turn, the thermodynamic SF requirement, that is $\left(S_{1} \geq 2 T_{1}\right)$, is fulfilled for pentacenes rendering this process exothermic and unidirectional (13). Finally, from SF in hexacenes two triplet excited states plus a certain amount of phonons are derived as the main relaxation products. In other words, the dissipated heat leads to a decrease in SF yields and rates (15).

In terms of exploiting SF for improving device performances, e.g., hybrid solar cells, it is necessary to efficiently dissociate correlated triplet pairs as they are formed, to overcome triplettriplet annihilation $(5,16)$. Rapid injection of electrons into fullerenes, perylene diimides, colloidal nanocrystals, semiconductor substrates, etc. suggests a viable strategy. If successful, two charge carriers might be produced per absorbed photon and the photocurrents of the device can reach external quantum efficiencies of more than $100 \%(5,17,18)$.

A provocative debate has been ignited about the mechanism of SF at the molecular level $(19,20)$. Controversy exists around the electronic states that are involved in the process, the coupling among them, and the effective nuclear dynamics (14, 21-27). Two contrasting SF mechanisms have been traditionally postulatedthe direct and the two-step mechanism. These mechanisms differ in the number and nature of the electronic states that are involved in the SF process. For the direct mechanism, the nonradiative relaxation of the initially populated bright state proceeds via a correlated triplet pair state of singlet character--sometimes called multiexcitonic (ME) state--which then dissociates into two separated triplet excited states (28). For the two-step mechanism, the relaxation of the bright state occurs via an intermediate charge transfer (CT) state to the ME state. As in the direct mechanism, the ME state eventually allows the two triplet excited states involved to separate and undergo separate spin relaxation $(11,21-23)$. Recent works have, however, challenged these traditional viewpoints on the SF mechanism and several new models have been proposed. In particular, it has been suggested $(13,29,30)$ that the initial excitation produces a coherent superposition of the lowest-lying absorbing state and the ME state, with the latter splitting into two separated triplet states after

\section{Significance}

In the present work, we show compelling evidence for the unprecedented intramolecular singlet fission at room temperature and in dilute solutions within a set of three different regioisomeric pentacene dimers. Pump-probe experiments, which were complemented by theoretical calculations using high-level ab initio multireference perturbation theory methods, corroborate triplet quantum yields as high as $156 \pm 5 \%$. To this end, electronic couplings between the two pentacenes in the dimers, by virtue of through-bond or through-space interactions, are decisive in tuning the rates of singlet fission.

Author contributions: M.T., R.R.T., and D.M.G. designed research; J.Z., D.L., P.B.C., E.T.C., R.C., and B.S.B. performed research; J.Z., P.B.C., E.T.C., R.C., B.S.B., and D.M.G. analyzed data; and M.T., R.R.T., and D.M.G. wrote the paper.

The authors declare no conflict of interest.

This article is a PNAS Direct Submission. D.R.R. is a guest editor invited by the Editorial Board.

${ }^{1}$ To whom correspondence may be addressed. Email: rik.tykwinski@fau.de or dirk.guldi@ fau.de.

This article contains supporting information online at www.pnas.org/lookup/suppl/doi:10. 1073/pnas.1422436112/-/DCSupplemental. 
decoherence. On the other hand, recent theoretical works $(14,27)$ have proposed a model for SF in dimers, in which the ME state is formed from the absorbing state via a superexchange mechanism involving CT states, although the kinetics of the process is onestep-like. All of the above suggests that the traditional classification of SF mechanisms as direct or two-step is presumably too simple to describe the complexity of the process.

Until recently, most studies regarding SF have been carried out in the solid state (11). However, in a groundbreaking report, Friend and coworkers showed that SF could be observed in solution at room temperature for a pentacene derivative (31). Key to this discovery was the formation of an intermediate state via the collision of a singlet excited-state pentacene and a second pentacene that was in the ground state. This study broke for the first time, to our knowledge, the dogma of molecular order as a mandate for SF and demonstrated that the order and packing might not be as crucial as believed, leaving SF as an intrinsic property even in a state of disorder.

In this present contribution, we report on intramolecular SF within a set of three different pentacene dimers (Fig. 1), reaching triplet quantum yields as high as $156 \pm 5 \%$, as established by means of pump-probe experiments in solution. Experimental results are complemented by theoretical calculations using highlevel ab initio multireference perturbation theory methods. In the series of pentacene dimers, the two pentacenes are linked via a phenylene spacer in an ortho-, meta-, and para-arrangement to impose geometrical control, which influences through-space and through-bond couplings in the ground- and excited state. The electronic coupling element, which governs SF and triplet-triplet annihilation, is mediated through-bond in the linearly and crossconjugated para- and meta-isomers, respectively. In the orthoisomer, through-space coupling should dominate due to the unique spatial proximity of the pentacenes $(32,33)$. Through experiments, we establish a sound picture of the SF mechanism in covalently linked pentacene dimers, showing the involvement of CT states.

\section{Results and Discussion}

Synthesis. Synthesis of the pentacene dimers was based on desymmetrization of 6,13-pentacenequinone to give building block 1 (Fig. 1) (34). Dimers $\boldsymbol{o - 2}, \boldsymbol{m}-\mathbf{2}$, and $\boldsymbol{p}-\mathbf{2}$ were then assembled to probe the effects of the geometry about the phenylene $\pi$-spacer. Sonogashira coupling of 1 with ortho-, meta-, and para-diiodobenzene afforded intermediates $\mathbf{3 - 5}$, respectively, in $\sim 70 \%$ yield. $\mathrm{SnCl}_{2}$-mediated reductive aromatization in the presence of aq. $\mathrm{H}_{2} \mathrm{SO}_{4}$ yielded finally dimers $\boldsymbol{o - 2}, \boldsymbol{m - 2}$, and $\boldsymbol{p} \mathbf{- 2}$. The orthophenylene-linked pentacene dimer $\boldsymbol{o - 2}$ has reasonable solubility in solvents such as $\mathrm{CH}_{2} \mathrm{Cl}_{2}, \mathrm{CHCl}_{3}$, and THF, whereas the corresponding meta- and para-derivatives, $\boldsymbol{m - 2}$ and $\boldsymbol{p}-2$ respectively, have poor solubility in these solvents.

Excited-State Energetics. Steady-state spectroscopies were used to examine the singlet- $\left(\mathrm{E}_{\text {Singlet }}\right)$ and triplet-state energetics $\left(\mathrm{E}_{\text {Triplet }}\right)$ of the pentacene dimers (SI Appendix, Figs. S14 and S15). First, long wavelength absorption maxima in the 660-678-nm range in toluene and 666-691-nm range in benzonitrile were determined, together with short wavelength fluorescence maxima at $664 \mathrm{~nm}$ $(\boldsymbol{m}-2), 676 \mathrm{~nm}(\boldsymbol{o}-2)$, and $670 \mathrm{~nm}(\boldsymbol{p}-2)$ and fluorescence quantum yields ranging from $0.5 \%$ to $1.5 \%$ in toluene (Table 1 ).

From the corresponding energetic differences we determined lowest-lying singlet absorbing-state energies of $1.84 \pm 0.05 \mathrm{eV}$ for the pentacene dimers $\boldsymbol{o - 2}, \boldsymbol{m - 2}$, and $\boldsymbol{p - 2}$. At first glance, all three dimers give rise to absorption patterns that resemble those of 6,13-bis(triisopropylsilylethynyl)pentacene (TPc; see SI Appendix for structure), including vibrational fine structure in toluene and in benzonitrile in the range from 558 to $691 \mathrm{~nm}$, although the absorptions of the dimers are red-shifted relative to TPc. A closer look at the ortho-dimer $\boldsymbol{o - 2}$ suggests, however, strong electronic couplings by means of through-space interactions in the ground state. This stems from the close spatial arrangement of the face-toface aligned pentacenes (Fig. 1) as seen in the optimized geometries (see SI Appendix for details). Finally, triplet excited-state energies of $0.77 \mathrm{eV}$ were derived for $\boldsymbol{o - 2}, \boldsymbol{m - 2}$, and $\boldsymbol{p}$-2 from maxima at $1,610 \mathrm{~nm}$ observed in phosphorescence measurements at $77 \mathrm{~K}$ (SI Appendix, Fig. S16). Thus, the singlet and triplet energies of pentacene dimers $\mathbf{2}$ fulfill the thermodynamic requirement for SF, that is, twice the triplet excitation energy should not exceed the lowest singlet excitation energy. Secondly, to elucidate a possible CT state, we probed $\boldsymbol{m - 2}$ by means of square wave voltammetry. In benzonitrile (SI Appendix, Fig. S17), where solvent stabilization is effective, we derived a first one-electron reduction of $-1.43 \mathrm{~V}$ and a first one-electron oxidation of $0.44 \mathrm{~V}$ (versus $\mathrm{Fc} / \mathrm{Fc}^{+}$). Neglecting any Coulombic attractive terms, the energy level of the intramolecular CT state for $\boldsymbol{m}-\mathbf{2}$ would have a lower limit of $1.87 \mathrm{eV}$ and is, thus, quite achievable during excitation. Please note that this intramolecular CT state places the one-electron reduced form on one pentacene and the one-electron oxidized form on the other pentacene, lowering attractive interactions and facilitating its formation.

Transient Absorption Spectroscopy and SF Kinetics. As a reference, TPc in Me-THF $\left(3.0 \times 10^{-4} \mathrm{M}\right)$ was examined in pump-probe experiments with an excitation wavelength of $656 \mathrm{~nm}$ to stimulate population of only vibrational states of the first singlet excited state (SI Appendix, Fig. S18). Additionally, the photon flux was adjusted to $6.6 \times 10^{9}$ photons per pulse to excite, on average, less than $10 \%$ of the ground-state TPc to rule out multiple excitations of a single molecule. The major deactivation pathway for photoexcited TPc includes a $12.3 \pm 0.1$-ns intersystem crossing of the singlet excited state to afford the corresponding triplet manifold. The corresponding triplet excited state deactivates with a rather long lifetime of $24.0 \pm 0.5 \mu$ s to reinstate the singlet ground state. In terms of the singlet excited-state characteristics, maxima at 447, 508, 538, 572, 845 , and $1,400 \mathrm{~nm}$ were noted, as well as minima at 595, 641, and $710 \mathrm{~nm}$. Notably, the feature at $710 \mathrm{~nm}$ corresponds to stimulated emission. The triplet excited state has maxima at 465 and $498 \mathrm{~nm}$ and minima at 546, 587, and $641 \mathrm{~nm}$ in the differential absorption

Table 1. Spectroscopic data of pentacene dimers

\begin{tabular}{|c|c|c|c|c|c|c|c|c|}
\hline Pentacene dimer & Solvent & $E_{\text {Singlet }}[\mathrm{eV}]$ & $E_{\text {Triplet }}[\mathrm{eV}]$ & $\Phi_{F}[\%]$ & $k_{C T}\left[\mathrm{~s}^{-1}\right]$ & $k_{S F}\left[\mathrm{~s}^{-1}\right]$ & $k_{T T A}\left[\mathrm{~s}^{-1}\right]$ & $\Phi_{\text {Triplet }}[\%]$ \\
\hline$m-2$ & Toluene & 1.88 & $0.77 *$ & $0.6 \pm 0.1$ & $3.97 \pm 0.80 \times 10^{10}$ & $1.11 \pm 0.10 \times 10^{10}$ & $3.85 \pm 0.15 \times 10^{8}$ & $125 \pm 5^{\dagger}$ \\
\hline & Benzonitrile & 1.86 & & $0.4 \pm 0.1$ & $6.10 \pm 0.70 \times 10^{10}$ & $1.59 \pm 0.15 \times 10^{10}$ & $4.55 \pm 0.15 \times 10^{8}$ & $156 \pm 5^{\dagger}$ \\
\hline $0-2$ & Toluene & 1.87 & - & $1.6 \pm 0.2$ & - & - & - & - \\
\hline$p-2$ & $\begin{array}{c}\text { Toluene } \\
\text { Benzonitrile }\end{array}$ & $\begin{array}{l}1.83 \\
1.79\end{array}$ & - & $\begin{array}{c}0.3 \pm 0.1 \\
-\end{array}$ & $-\frac{}{3.70+1.5}$ & $0 \times 10^{11 \mp}$ & $5.78+\overline{0.50} \times 10^{10}$ & $\frac{-}{130+10}$ \\
\hline
\end{tabular}

Singlet excited-state energy $\left(E_{\text {Singlet }}\right)$, triplet excited-state energy $\left(E_{\text {Triplet }}\right)$, fluorescence quantum yields $\left(\Phi_{F}\right)$, rate constants for charge transfer $\left(k_{C T}\right)$, rate constants for triplet growth $\left(k_{S F}\right)$, rate constants for triplet decay $\left(k_{T T A}\right)$, triplet excited-state quantum yields $\left(\Phi_{T r i p l e t}\right)$ for $\boldsymbol{m}-\mathbf{2}, \mathbf{o - 2}$, and $\boldsymbol{p}-\mathbf{2}$ in toluene and benzonitrile.

*Determined in frozen Me-THF at $77 \mathrm{~K}$

${ }^{\dagger}$ Determined by using the power-dependent method.

${ }^{\text {F}}$ The short-lived nature of the triplet excited states in $\mathbf{0 - 2}$ hampers a meaningful triplet excited-state quantum yield determination. 

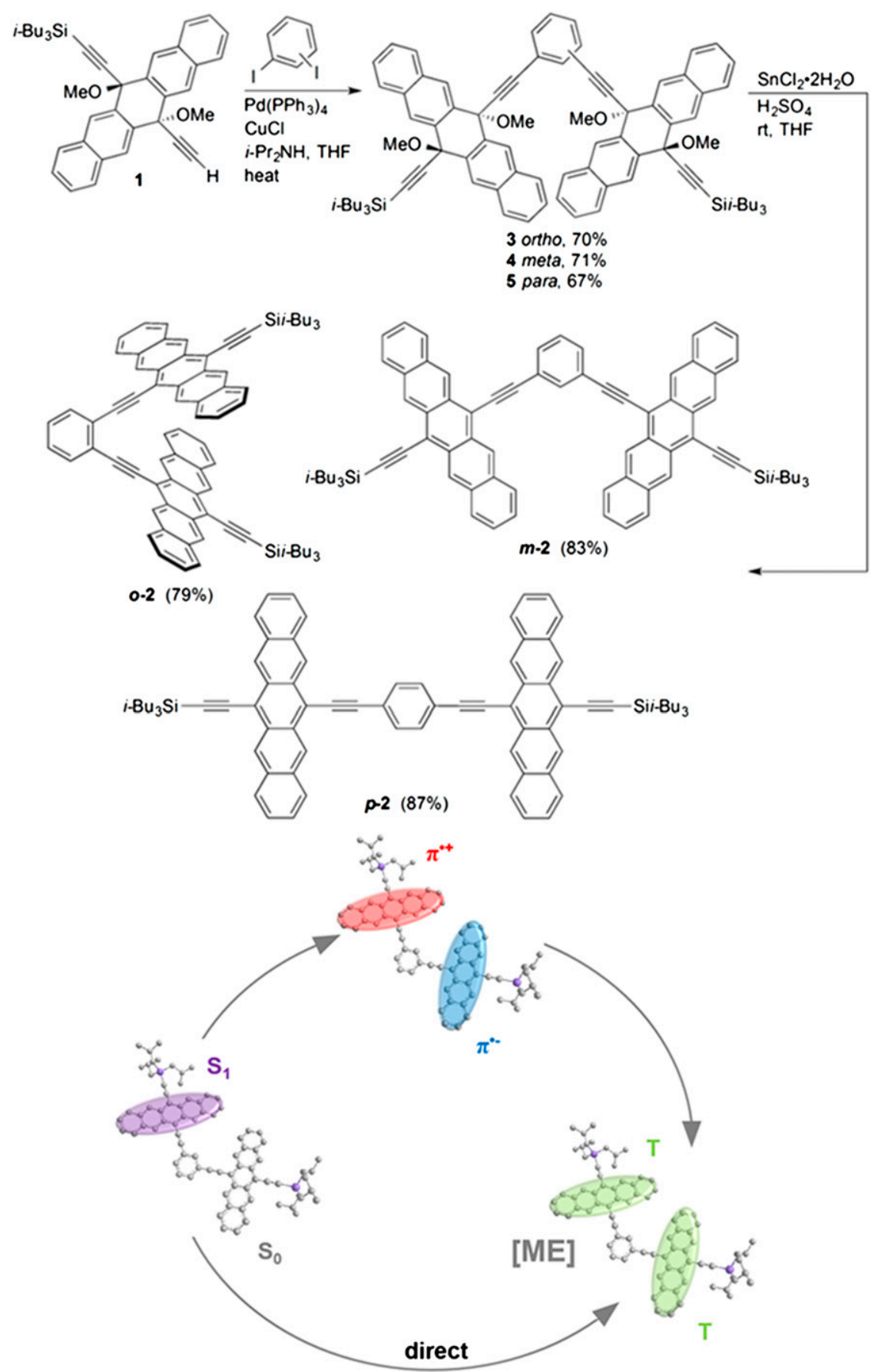

Fig. 1. (Upper) Synthesis of pentacene dimers $\mathbf{o - 2}, \mathbf{m}-\mathbf{2}$, and $\mathbf{p - 2}$. (Lower) SF mechanism.

spectra. All of these observations are in excellent agreement with previously reported singlet and triplet excited-state absorptions of TPc in solution (31). When using singlet molar extinction coefficients of $8.3 \times 10^{4} \mathrm{M}^{-1} \cdot \mathrm{cm}^{-1}$ at the $447-\mathrm{nm}$ maximum, in combination with triplet molar extinction coefficients of $1.45 \times$ $10^{5} \mathrm{M}^{-1} \cdot \mathrm{cm}^{-1}$ at the 498-nm maximum, the computed TPc triplet quantum yield is $16 \%$. For this analysis, ground-state extinction coefficients of $3.3 \times 10^{4} \mathrm{M}^{-1} \cdot \mathrm{cm}^{-1}$ at the $641-\mathrm{nm}$ minimum and $1.6 \times 10^{4} \mathrm{M}^{-1} \cdot \mathrm{cm}^{-1}$ at the $587-\mathrm{nm}$ minimum were used.

Next, the three pentacene regioisomers $\boldsymbol{o - 2}, \boldsymbol{m - 2}$, and $\boldsymbol{p}-\mathbf{2}$ were examined in solvents of varied polarity (i.e., toluene, THF, and benzonitrile) with photoexcitation at either 610 or $656 \mathrm{~nm}$ as shown in Fig. 2 as well as SI Appendix, Figs. S19, and S20, respectively, and the same low photon flux described for TPc-vide supra. The meta-isomer $(\boldsymbol{m}$-2) was examined in benzonitrile and at time delays as short as $0.5 \mathrm{ps}$ singlet excited-state features are discernible in the form of maxima at 456, 510, 582, and $670 \mathrm{~nm}$. In contrast with the slow intersystem crossing seen for TPc, these singlet excited-state features are rapidly replaced by strong absorptions, which are concentration independent in the range from $1.0 \times 10^{-5}$ to $1.0 \times 10^{-4} \mathrm{M}$ and, thus, strictly unimolecular. These strong absorptions, which appear at time delays of around $200 \mathrm{ps}$, bear great resemblance with that of the triplet excited state of $\boldsymbol{m}$-2. In particular, maxima at $477,510,855$, and 972 are complemented by minima at 610 and $664 \mathrm{~nm}$ and are a perfect match to the photosensitized triplet excited-state spectra of the dimer $\boldsymbol{m - 2}$ (vide infra). 

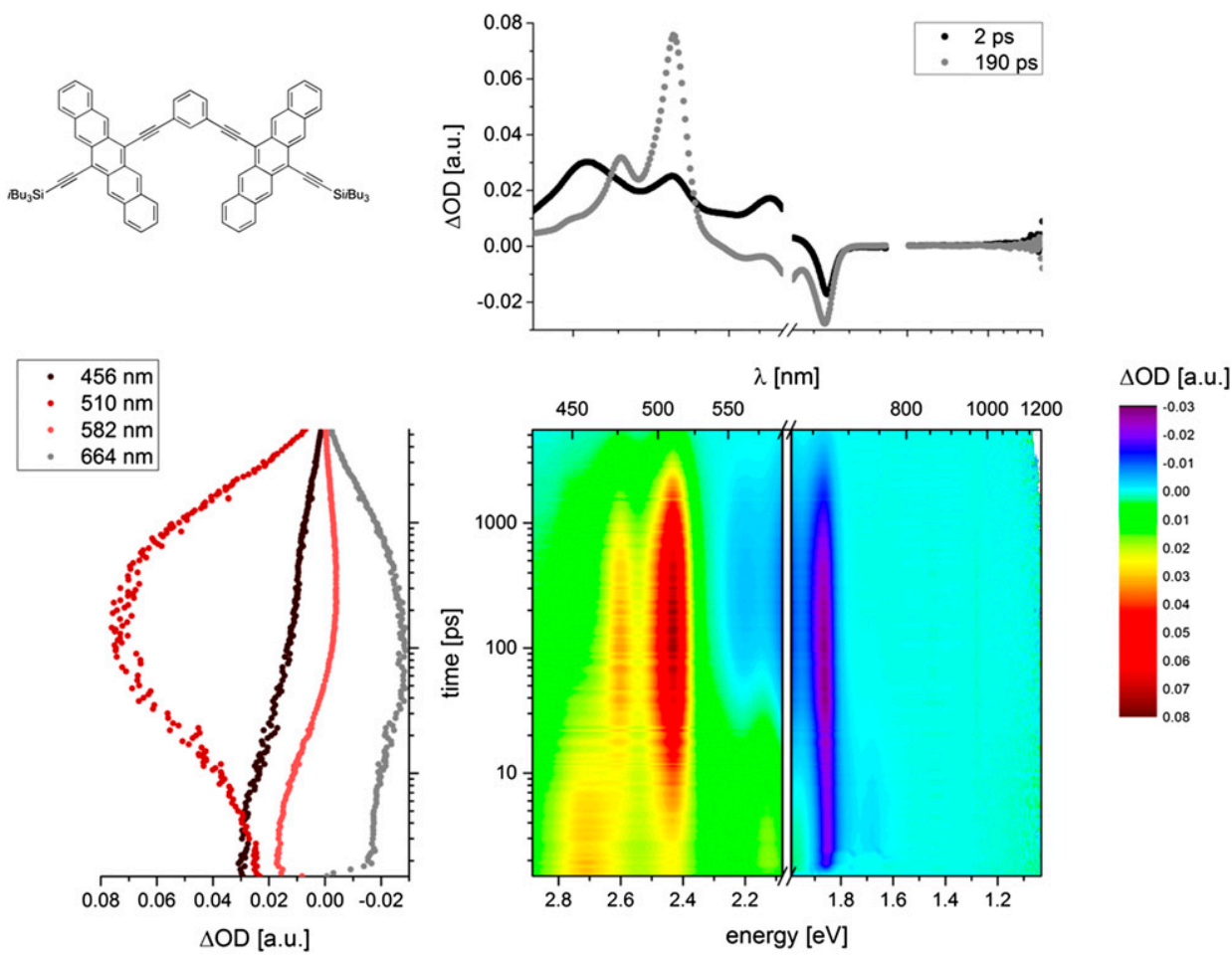

Fig. 2. $S F$ in pentacene dimer $\mathbf{m}-\mathbf{2}$. (Upper Left) Chemical structure. (Upper Right) Differential absorption spectra (visible and near-infrared) of the spectra shown in the lower right with time delays of $2.0 \mathrm{ps}$ (black) and $190.0 \mathrm{ps}$ (gray). (Lower Left) Time absorption profiles of the spectra shown in the lower right at $456 \mathrm{~nm}$ (black), $510 \mathrm{~nm}$ (red), $582 \mathrm{~nm}$ (orange), and $664 \mathrm{~nm}$ (gray) illustrating the dynamics of the singlet excited-state formation followed by the singlet to triplet transformation in the form of SF, and triplet-triplet annihilation. (Lower Right) Differential absorption changes (visible and near-infrared) obtained upon femtosecond pump-probe experiments $(610 \mathrm{~nm})$ of $\boldsymbol{m}-\mathbf{2}\left(3.5 \times 10^{-5} \mathrm{M}\right)$ in argon-saturated benzonitrile at room temperature with several time delays between 0 and 5,500 ps.

A particularly striking effect observed for $\boldsymbol{m}-\mathbf{2}$ during the accelerated triplet excited-state formation is the long wavelength blue shift, from 670 to $664 \mathrm{~nm}$, that is accompanied by a $155 \pm$ $10 \%$ intensification of the transient bleaching. This effect is notable upon $610-\mathrm{nm}$ excitation rather than 656-nm excitation. If the ground-state absorption depletion in this spectral region were quantitative for the singlet excited state, this infers more than one triplet excited state is formed per dimer. Nevertheless, the triplet excited state is surprisingly short-lived and decays with concentration-independent kinetics. The only plausible modus operandi is based on intramolecular triplet-triplet annihilation governed by the unique scenario that more than one triplet excited state is localized on each dimer. Multiwavelength analyses attest that the triplet excited-state formation is biexponential in benzonitrile, and it features two distinct steps with CT $\left(\tau_{C T}\right)$ and SF $\left(\tau_{S F}\right)$ lifetimes of $16.4 \pm 1.9$ and $63.0 \pm 6.3$ ps, respectively. From the study of solvent dependence vide infra, we postulate the involvement of an additional state that possesses an intramolecular CT character and mediates the transition to the ME state (either directly or virtually). A closer look at the transient absorption spectra taken at delay times of 80-300 ps reveals a 445-nm shoulder, which is likely to stem from the CT state (SI Appendix, Fig. S21). Once formed, the triplet excited-state decay is monoexponential for $\boldsymbol{m - 2}$, with a rather short triplet-triplet annihilation lifetime $\left(\tau_{T T A}\right)$ of $2.2 \pm 0.1 \mathrm{~ns}$.

Turning our attention to the para- and ortho-isomers ( $p-2$ and $o-$ $2)$, their singlet excited-state features are discernible immediately after photoexcitation in benzonitrile. For $\boldsymbol{p}$-2, these are maxima at 462 and 1,117 nm and a minimum at $686 \mathrm{~nm}$ (SI Appendix, Fig. S19). The features of the singlet state of $\boldsymbol{p - 2}$, however, transform even faster than in $\boldsymbol{m} \mathbf{- 2}$ into the triplet excited-state maxima at 475 and $508 \mathrm{~nm}$ as well as its minimum at $670 \mathrm{~nm}$. From multiwavelength analyses we derive lifetimes for the growth and decay of the triplet excited state as $2.7 \pm 1.0$ and $17.3 \pm 1.3 \mathrm{ps}$, respectively. It is likely that the dramatic difference in behavior in $\boldsymbol{p}$-2 versus $\boldsymbol{m}$-2 kinetics derives from enhanced through-bond electronic coupling in the ground state of the para-isomer as a means to impact both SF and triplet-triplet annihilation $(32,33)$. As such, the electronic coupling matrix elements as the decisive factor to govern CT are larger in $\boldsymbol{p}-\mathbf{2}$ than in $\boldsymbol{m - 2}$ (32). For $\boldsymbol{o - 2}$, the 472- and 507-nm fingerprints for the singlet and triplet excited states, respectively, evolve in pump-probe experiments. Here, in contrast with $\boldsymbol{p}$-2 and $\boldsymbol{m}-\mathbf{2}$, strong through-space couplings between the two pentacene moieties affect the triplet excited-state kinetics-both in terms of formation and decay-and deconvolution results in upper values of $0.5 \pm 0.2 \mathrm{ps}$ and $12.0 \pm 0.3 \mathrm{ps}$, for formation and decay, respectively. The short-lived nature of the triplet excited states in $\boldsymbol{p - 2}$ and $\boldsymbol{o - 2}$ hampers a meaningful triplet excited-state quantum yield determination. The latter is corroborated in the geometry-optimized structures (SI Appendix, Fig. S27), which reveal, on one hand, the coplanarity between the two pentacenes in $\boldsymbol{p}-\mathbf{2}$ and $\mathbf{m - 2}$, and, on the other hand, only sizable through-space couplings in $\boldsymbol{o}-\mathbf{2}$.

Triplet Quantum Yield and SF Yield Determination. Using the differential absorption changes at 610 and $664 \mathrm{~nm}$ for $\boldsymbol{m} \mathbf{- 2}$, for which ground-state extinction coefficients of $2.8 \times 10^{4}$ and $5.3 \times$ $10^{4} \mathrm{M}^{-1} \cdot \mathrm{cm}^{-1}$ have been determined, the singlet and triplet molar extinction coefficients are calculated as $7.9 \times 10^{4}$ and $1.4 \times$ $10^{5} \mathrm{M}^{-1} \cdot \mathrm{cm}^{-1}$, respectively. In recent studies, it was hypothesized that the bleaching at $610 \mathrm{~nm}$ is elusive for the triplet excited state, whereas that the bleaching at $664 \mathrm{~nm}$ serves as an internal standard to calibrate the singlet excited state (31). Because the meta-dimer $\boldsymbol{m}-\mathbf{2}$ has only very weak ground-state absorption at $456 \mathrm{~nm}$, the observed amplitude of differential absorption at 456 $\mathrm{nm}$ and $0.5 \mathrm{ps}$ relates entirely to the singlet excited-state concentration formed after excitation. In doing so, differential absorption changes of 0.028 a.u. at $456 \mathrm{~nm}$ and 0.0726 a.u. at $510 \mathrm{~nm}$ were converted into a triplet quantum yield $\left(\Phi_{\text {Triplet }}\right)$ of $145 \pm 10 \%$. This high triplet quantum yield along with its ultrafast formation provides strong evidence that the triplet excited state for $\boldsymbol{m}-\mathbf{2}$ forms by intramolecular SF between the two pentacenes. The triplet quantum yield for $\boldsymbol{p}-\mathbf{2}$ was determined by extrapolation to time zero as $130 \%$.

Taking the ratio from $510 / 456 \mathrm{~nm}$ as an indicator for the respective quantum yield, we note that it increases from toluene $(126 \pm 3 \%)$ to THF $(132 \pm 2 \%)$ and to benzonitrile $(145 \pm 10 \%)$. We surmise from this trend that the mediating step, which dictates the triplet excited-state formation, features CT-type character and this operates through-bond for dimer $\boldsymbol{m}$-2. Such a postulate fits well 
with the fact that the triplet excited-state kinetic growth is biexponential and that the kinetics are solvent dependent. Biexponential fits of these data, for example, yields lifetimes of $25.2 \pm$ 4.8/90.2 $\pm 9.0 \mathrm{ps}$ in toluene, $18.6 \pm 2.9 / 70.3 \pm 7.0 \mathrm{ps}$ in THF, and $16.4 \pm 1.9 / 63.0 \pm 6.3 \mathrm{ps}$ in benzonitrile. In contrast, the intramolecular triplet-triplet annihilation lacks any appreciable solvent polarity dependence as evidenced by nearly constant lifetimes of $2.6 \pm 0.1 \mathrm{~ns}$ in toluene, $2.5 \pm 1.0 \mathrm{~ns}$ in THF, and $2.2 \pm 0.1 \mathrm{~ns}$ in benzonitrile.

Alternatively, the power-dependent method using the differential absorption changes at 670 and $664 \mathrm{~nm}$ was used to calculate the transient extinction coefficient of the bleaching as $5.7 \times 10^{4}$ and $8.3 \times 10^{4} \mathrm{M}^{-1} \cdot \mathrm{cm}^{-1}$ for the singlet and the triplet excited state, respectively. Consequently, we obtained a triplet quantum yield for the meta-dimer $(\boldsymbol{m}-2)$ of $156 \pm 5 \%$. The main advantage of this method is that it allows the determination of transient extinction coefficients correlated by the amount of excited molecules in the exact range of excitation without using an external reference (31).

To complement the power-dependent studies, we also generated the triplet excited-state signatures of all three dimers by means of triplet sensitization experiments in toluene. In these experiments, an $N$-methylfulleropyrrolidine ( $N$-MFP; see $S I$ Appendix for structure) with a triplet quantum yield close to unity and a triplet excited-state energy of $1.5 \mathrm{eV}$ was taken and selectively excited at $480 \mathrm{~nm}$, which coincides with a minimum in the pentacene ground-state absorption. $N$-MFP $\left(8.0 \times 10^{-5} \mathrm{M}\right)$ was photoexcited, leading to the sequential formation of its short-lived singlet and its long-lived triplet excited states with their 695- and 900-nm fingerprint absorptions, respectively. In the presence of the three pentacene regioisomers $\boldsymbol{o - 2}, \boldsymbol{m}-\mathbf{2}$, and $p$-2 as well as TPc in concentration regimes from $1.0 \times 10^{-5}$ to $1.0 \times$ $10^{-4} \mathrm{M}$, a set of newly developing transient absorption features evolves with the depletion of the $N$-MFP triplet excited state (Fig. 3 as well as SI Appendix, Figs. S22 and S23). The metadimer $\boldsymbol{m}$-2, for example, shows a transient maximum at $506 \mathrm{~nm}$ and minima at 559, 605, and $661 \mathrm{~nm}$. While for TPc pentacene, a 503-nm maximum as well as 547-, 592-, and 643-nm minima were noted, for $\boldsymbol{o - 2}$ and $\boldsymbol{p}-\mathbf{2}, 512 / 507-\mathrm{nm}$ maxima as well as 570-, 614-, 664-nm and 562-, 614-, and 668-nm minima, respectively, were recorded. Importantly, a closer look reveals that the decay and growth are pentacene concentration dependent. Complementary analyses of the $N$-MFP triplet excited-state decay as well as of the pentacene triplet excited-state growth provide the means to derive the second-order energy transfer rate constants ( $S I$ Appendix, Fig. S24). The rate constants in deoxygenated toluene are $1.6 \pm 0.1 \times 10^{9} \mathrm{M}^{-1} \cdot \mathrm{s}^{-1}$ for $\boldsymbol{o - 2}, 3.4 \pm 0.3 \times 10^{9} \mathrm{M}^{-1} \cdot \mathrm{s}^{-1}$ for $\boldsymbol{m}$-2, and $8.8 \pm 0.5 \times 10^{9} \mathrm{M}^{-1} \cdot \mathrm{s}^{-1}$ for $\boldsymbol{p}$-2, which infers that diffusion controls the transduction of triplet excited-state energy. Once formed, the pentacene triplet excited states in $\boldsymbol{o - 2}, \boldsymbol{m - 2}$, and $\boldsymbol{p - 2}$ are subject to concentration-dependent deactivations via efficient intermolecular triplet-triplet annihilation with lifetimes in the range of tens of microseconds. Intramolecular contributions under these conditions play no major role. A rate constant of $1.5 \pm$ $0.5 \times 10^{8} \mathrm{M}^{-1} \cdot \mathrm{s}^{-1}$ was derived for $\boldsymbol{m}-\mathbf{2}$ from pseudo-first-order analyses (SI Appendix, Fig. S25). From Figs. 2 (i.e., direct excitation experiments) and 3 (i.e., triplet sensitization experiments) the singlet molar extinction coefficient at $456 \mathrm{~nm}$, the triplet molar extinction coefficient at $506 \mathrm{~nm}$, and the triplet quantum yield were determined for $\boldsymbol{m - 2}$ as $7.4 \times 10^{4} \mathrm{M}^{-1} \cdot \mathrm{cm}^{-1}, 1.5 \times 10^{5} \mathrm{M}^{-1} \cdot \mathrm{cm}^{-1}$, and $130 \pm 10 \%$, respectively (see SI Appendix for details).

Theory. To characterize the electronic states involved in the SF process, we have used the second-order complete active space perturbation theory with a complete active space self-consistent field reference wavefunction (CASPT2/CASSCF) approach (see SI Appendix for details). This ab initio multireference perturbation theory method is known to provide accurate results for electronic states of very different chemical character like those involved in SF (35). The results, depicted in SI Appendix, Table S1, reveal a set of closely lying electronic excited states of ME and locally excited (LE) character. Whereas in $\boldsymbol{m}-\mathbf{2}$ and $\boldsymbol{p}-\mathbf{2}$ the first excited state, $S_{1}$, is of ME character and $S_{2}$ and $S_{3}$ are of LE character, respectively, this order changes in $\boldsymbol{o - 2}$. In all cases, however, the ME state is quasi-degenerate with the initially excited LE states and, therefore, a direct pathway for SF is energetically possible. The results obtained in vacuo predict CT states located at significantly higher energies than the LE and ME states. Additional calculations using the polarization continuum model (see SI Appendix for details) for benzonitrile reveal a
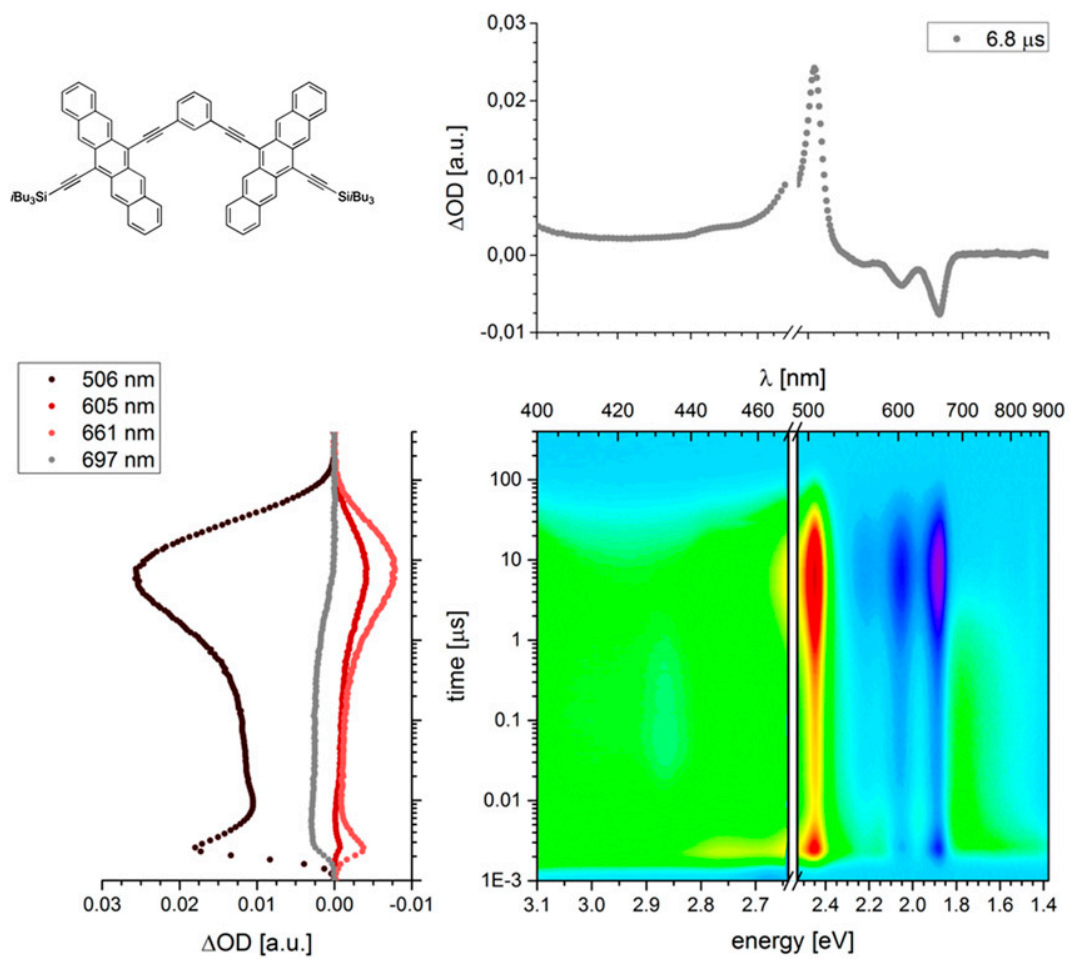

Fig. 3. Triplet-triplet sensitization of pentacene dimer $\mathbf{m}$-2. (Upper Left) Chemical structure. (Upper Right) Differential absorption spectrum (visible and nearinfrared) of the spectra shown in the lower right with a time delay of $6.8 \mu \mathrm{s}$ representing the triplet excited state of $\boldsymbol{m} \mathbf{- 2}$. (Lower Left) Time absorption profiles of the spectra shown in the lower right at $506 \mathrm{~nm}$ (black), $605 \mathrm{~nm}$ (red), $661 \mathrm{~nm}$ (orange), and $697 \mathrm{~nm}$ (gray) illustrating the dynamics of the N-MFP singlet excited-state formation followed by the intersystem crossing to the corresponding N-MFP triplet excited state and transduction of triplet excitedstate energy to $\mathbf{m}-\mathbf{2}$. (Lower Right) Differential absorption changes (visible and near-infrared) obtained upon femtosecond pump-probe experiments $(480 \mathrm{~nm}$ ) of $\mathrm{N}$-MFP $\left(8.0 \times 10^{-5} \mathrm{M}\right)$ and $m-2\left(1.0 \times 10^{-4} \mathrm{M}\right)$ in argonsaturated toluene at room temperature with several time delays between 0 and $400 \mu \mathrm{s}$. 
pronounced stabilization of the CT states which locates them $\sim 0.35 \mathrm{eV}$ above the lowest-lying LE and ME states in $\boldsymbol{m}-\mathbf{2}$ (SI Appendix). Taking into account the experimentally observed solvent dependence of the triplet yield and the kinetics, our results indicate that CT states participate in SF either as virtual states via a superexchange mechanism (14) or directly if nuclear relaxation of the lowest-lying absorbing state facilitates population of the CT state via internal conversion. In addition to the characterization of the excited singlet states, we also have characterized the excited triplet states of $\boldsymbol{m} \mathbf{- 2}$. The results in SI Appendix, Table S3 reveal a strong $\mathrm{T}_{1} \rightarrow \mathrm{T}_{9}$ excitation at $2.43 \mathrm{eV}$, which corresponds to the pronounced triplet feature at $510 \mathrm{~nm}$ observed in the transient absorption spectra (Fig. 3).

\section{Conclusions}

In conclusion, we used pump-probe experiments and complementary theoretical calculations using multireference perturbation theory to corroborate for the first time, to our knowledge, intramolecular SF in pentacene dimers. Three regioisomeric pentacene dimers, in which the two pentacenes are linked via a phenylene spacer in ortho-, meta, or para-position-o-2, $\mathbf{m - 2}$, and $\boldsymbol{p}$-2-feature triplet quantum yields as high as $156 \pm 5 \%$ mediated either by through-space or through-bond electronic couplings in the ground- and excited state. As recently hypothesized, mixing between the singlet excited state and the CT state might be helpful in terms of overcoming the activation barrier (14). It is in $\boldsymbol{m}$-2 where the right balance between triplet formation and triplet decay optimizes the SF yield. Such an efficient SF reaches well beyond the $3 \%$ mark found in tetracene dimers, for which, incidentally, it is the para-isomer that revealed the highest SF yields (7). The demonstrated depletion of the ground state, the quantitative analysis of forming more than one triplet excited state per singlet, and the very low concentrations used corroborate the intramolecular nature of SF. Although our studies demonstrated that the singlet excited states in all three different

1. Smith MB, Michl J (2010) Singlet fission. Chem Rev 110(11):6891-6936.

2. Shockley W, Queisser HJ (1961) Detailed balance limit of efficiency of p-n junction solar cells. J Appl Phys 32(3):510-519.

3. Ehrler B, Musselman KP, Böhm ML, Friend RH, Greenham NC (2012) Hybrid pentacene/asilicon solar cells utilizing multiple carrier generation via singlet exciton fission. Appl Phys Lett 101(15):153507.

4. Jadhav PJ, et al. (2012) Triplet exciton dissociation in singlet exciton fission photovoltaics. Adv Mater 24(46):6169-6174.

5. Lee J, Jadhav P, Baldo MA (2009) High efficiency organic multilayer photodetectors based on singlet exciton fission. Appl Phys Lett 95(3):033301.

6. Reusswig PD, Congreve DN, Thompson NJ, Baldo MA (2012) Enhanced externa quantum efficiency in an organic photovoltaic cell via singlet fission exciton sensitizer. Appl Phys Lett 101(11):113304.

7. Müller AM, Avlasevich YS, Schoeller WW, Müllen K, Bardeen CJ (2007) Exciton fission and fusion in bis(tetracene) molecules with different covalent linker structures. J Am Chem Soc 129(46):14240-14250.

8. Johnson JC, Reilly TH, III, Kanarr AC, van de Lagemaat J (2009) The ultrafast photophysics of pentacene coupled to surface plasmon active nanohole films. J Phys Chem $C$ 113(16):6871-6877.

9. Marciniak H, Pugliesi I, Nickel B, Lochbrunner S (2009) Ultrafast singlet and triplet dynamics in microcrystalline pentacene films. Phys Rev $B$ 79(23):235318.

10. Wilson MW, et al. (2011) Ultrafast dynamics of exciton fission in polycrystalline pentacene. J Am Chem Soc 133(31):11830-11833.

11. Yost SR, et al. (2014) A transferable model for singlet-fission kinetics. Nat Chem 6(6): 492-497.

12. Bayliss SL, et al. (2014) Geminate and nongeminate recombination of triplet excitons formed by singlet fission. Phys Rev Lett 112(23):238701.

13. Chan WL, Ligges M, Zhu XY (2012) The energy barrier in singlet fission can be overcome through coherent coupling and entropic gain. Nat Chem 4(10):840-845.

14. Berkelbach TC, Hybertsen MS, Reichman DR (2013) Microscopic theory of singlet exciton fission. II. Application to pentacene dimers and the role of superexchange. J Chem Phys 138(11):114103.

15. Busby $E$, et al. (2014) Multiphonon relaxation slows singlet fission in crystalline hexacene. J Am Chem Soc 136(30):10654-10660.

16. Rao A, et al. (2010) Exciton fission and charge generation via triplet excitons in pentacene/ $C_{60}$ bilayers. J Am Chem Soc 132(36):12698-12703.

17. Congreve DN, et al. (2013) External quantum efficiency above $100 \%$ in a singletexciton-fission-based organic photovoltaic cell. Science 340(6130):334-337.

18. Eaton SW, et al. (2013) Singlet exciton fission in polycrystalline thin films of a slipstacked perylenediimide. J Am Chem Soc 135(39):14701-14712. pentacene dimers are rapidly and efficiently converted to triplet excited states via SF, this is considered only as a preliminary step toward realizing SF enhanced devices. In this context, a sound platform en route toward charge separation with suitable electron acceptors and charge collection at suitable electrodes is forming.

\section{Materials and Methods}

The synthesis of $\mathbf{o - 2}, \boldsymbol{m}-\mathbf{2}$, and $\mathbf{p - 2}$, materials, general methods, and computational details are described in SI Appendix.

For the photophysical characterization (see SI Appendix for details), the samples were placed in fluorometric cuvettes with different pathways and, when necessary, purged with argon. Steady-state UV-vis absorption spectra were acquired at room temperature (RT) using a Perkin Elmer Lambda 2 spectrometer. Steady-state fluorescence spectra were carried out at a FluoroMax3 spectrometer from Horiba in the visible detection range (RT) and at a FluoroLog3 spectrometer from Horiba in the near infrared. Femtosecond ( $\mathrm{fs}$ ) transient absorption experiments were carried out with an amplified Ti:Sapphire CPA-2110 fs laser system (Clark MXR: output $775 \mathrm{~nm}, 1 \mathrm{kHz}, 150$-fs pulse width) using transient absorption pump/probe detection systems (Helios and Eos, Ultrafast Systems).

Details of the calculations can be found in the SI Appendix. Briefly, the geometries of $\boldsymbol{o - 2}, \boldsymbol{m}-\mathbf{2}$, and $\boldsymbol{p}-\mathbf{2}$ were optimized using density functional theory. In all systems, $i-\mathrm{Bu}_{3} \mathrm{Si}-$ groups have been modelled using $\mathrm{H}_{3} \mathrm{Si}-$ to ensure computational tractability. For the different systems investigated vertical excitation energies were calculated at the CASPT2/CASSCF/ANO-SVDZP level of theory $(35,36)$. In all calculations, an active space of 8 electrons in 8 orbitals (comprising the HOMO-1, HOMO, LUMO, and LUMO+1 orbitals per pentacene unit; see SI Appendix, Fig. S26) was used.

ACKNOWLEDGMENTS. Generous allocation of computing time at the computing centers Erlangen (Regionales Rechenzentrum Erlangen), Munich (Leibniz-Rechenzentrum der Bayerischen Akademie der Wissenschaften in München), and Jülich (Jülich Supercomputing Centre) is gratefully acknowledged. Funding is gratefully acknowledged from the Emerging Fields initiative "Singlet Fission" supported by Friedrich-Alexander-Universität Erlangen-Nürnberg, as well as the Cluster of Excellence Engineering of Advanced Materials and "Solar Technologies Go Hybrid" - an initiative of the Bavarian State Ministry for Science, Research, and Art.

19. Zimmerman PM, Musgrave CB, Head-Gordon M (2013) A correlated electron view of singlet fission. Acc Chem Res 46(6):1339-1347.

20. Johnson JC, Nozik AJ, Michl J (2013) The role of chromophore coupling in singlet fission. Acc Chem Res 46(6):1290-1299.

21. Feng $X$, Luzanov AV, Krylov Al (2013) Fission of entangled spins: An electronic structure perspective. J Phys Chem Lett 4(22):3845-3852.

22. Parker SM, Seideman T, Ratner MA, Shiozaki T (2014) Model Hamiltonian analysis of singlet fission from first principles. J Phys Chem C 118(24):12700-12705.

23. Piland GB, Burdett JJ, Dillon RJ, Bardeen CJ (2014) Singlet fission: From coherences to kinetics. J Phys Chem Lett 5(13):2312-2319.

24. Teichen PE, Eaves JD (2012) A microscopic model of singlet fission. J Phys Chem B 116(37):11473-11481.

25. Zeng T, Hoffmann R, Ananth N (2014) The low-lying electronic states of pentacene and their roles in singlet fission. J Am Chem Soc 136(15):5755-5764.

26. Zimmerman PM, Bell F, Casanova D, Head-Gordon M (2011) Mechanism for singlet fission in pentacene and tetracene: From single exciton to two triplets. J Am Chem Soc 133(49): 19944-19952.

27. Berkelbach TC, Hybertsen MS, Reichman DR (2013) Microscopic theory of singlet ex citon fission. I. General formulation. J Chem Phys 138(11):114102.

28. Chan WL, et al. (2011) Observing the multiexciton state in singlet fission and ensuing ultrafast multielectron transfer. Science 334(6062):1541-1545.

29. Chan WL, et al. (2013) The quantum coherent mechanism for singlet fission: Experiment and theory. Acc Chem Res 46(6):1321-1329.

30. Akimov AV, Prezhdo OV (2014) Nonadiabatic dynamics of charge transfer and singlet fission at the pentacene/ $C_{60}$ interface. J Am Chem Soc 136(4):1599-1608.

31. Walker BJ, Musser AJ, Beljonne D, Friend RH (2013) Singlet exciton fission in solution. Nat Chem 5(12):1019-1024.

32. Gaab KM, Thompson AL, Xu J, Martínez TJ, Bardeen CJ (2003) Meta-conjugation and excited-state coupling in phenylacetylene dendrimers. J Am Chem Soc 125(31):9288-9289.

33. Quardokus RC, et al. (2012) Through-bond versus through-space coupling in mixedvalence molecules: Observation of electron localization at the single-molecule scale. J Am Chem Soc 134(3):1710-1714.

34. Lehnherr D, Murray AH, McDonald R, Tykwinski RR (2010) A modular synthetic approach to conjugated pentacene di-, tri-, and tetramers. Angew Chem Int Ed 49(35):6190-6194.

35. Roos BO, Fülscher MP, Malmqvist P-Å, Merchán M, Serrano-Andrés L (1996) Multiconfigurational perturbation theory: Applications in electronic spectroscopy. Advances in Chemical Physics: New Methods in Computational Quantum Mechanics, eds Prigogine I, Rice SA (John Wiley \& Sons, Hoboken, NJ), Vol 93, pp 219-331.

36. Pierloot K, Dumez B, Widmark P-O, Roos B (1995) Density matrix averaged atomic natural orbital (ANO) basis sets for correlated molecular wave functions. Theoret Chim Acta 90(2-3):87-114 